\title{
Subdividing the BeAT: Auditory And Motor Contributions to SynCHronization
}

\section{Janeen D. Loehr and Caroline Palmer McGill University, Montreal, Canada}

THE CURRENT STUDY EXAMINED HOW AUDITORY AND kinematic information influenced pianists' ability to synchronize musical sequences with a metronome. Pianists performed melodies in which quarter-note beats were subdivided by intervening eighth notes that resulted from auditory information (heard tones), motor production (produced tones), both, or neither. Temporal accuracy of performance was compared with finger trajectories recorded with motion capture. Asynchronies were larger when motor or auditory sensory information occurred between beats; auditory information yielded the largest asynchronies. Pianists were sensitive to the timing of the sensory information; information that occurred earlier relative to the midpoint between metronome beats was associated with larger asynchronies on the following beat. Finger motion was influenced only by motor production between beats and indicated the influence of other fingers' motion. These findings demonstrate that synchronization accuracy in music performance is influenced by both the timing and modality of sensory information that occurs between beats.

Received September 1, 2008, accepted February 18, 2009.

Key words: synchronization, timing, finger motion, music performance, auditory feedback

$\mathrm{M}$ OST SENSORIMOTOR SYNCHRONIZATION research focuses on the synchronization of simple movements (finger taps) with sequences ranging from simple (such as isochronous tones) to complex (such as rhythms; see Repp, 2005, 2006, for reviews). Relatively little is known, however, about how complex sequences such as musical melodies are synchronized. Music ensemble performance often requires synchrony among performers who produce different rhythms (see Goebl \& Palmer, 2009). Each performer must synchronize temporal intervals that are subdivided by those of other performers, and vice versa. Those subdivisions give rise to additional auditory and motor information, which could influence performers' ability to synchronize. The current study addressed the role of sensory information from subdivisions in synchronized music performance. Does hearing tones or producing movements between synchronized tones influence pianists' ability to synchronize melodies with a metronome?

When nonmusicians tap along with an isochronous auditory pacing sequence, their taps precede the pacing tones by 20 to $80 \mathrm{~ms}$ on average (Aschersleben, 2002). The tendency for taps to precede the pacing tones has been termed the mean negative asynchrony (MNA) and is smaller in musicians than nonmusicians (Aschersleben, 2002). The presence of additional tones between tones of the pacing sequence reduces the MNA, whether these tones evenly subdivide the pacing intervals (as in 1:n tapping, when participants tap with every $n$th tone of the pacing sequence; Repp, 2003), are timed randomly with respect to the pacing sequence (Wohlschläger \& Koch, 2000), or are part of a complex rhythm (as in synchronizing with music; Thaut, Rathbun, \& Miller, 1997). The MNA also is reduced when participants produce movements between synchronized taps. In $n: 1$ tapping, in which participants produce $n$ taps for every tone of the pacing sequence, asynchronies decrease as the number of taps between synchronized taps increases from two to three (Pressing, 1998). Asynchronies also are reduced when participants produce non-contact movements between taps. Wohlschläger and Koch (2000) recorded finger motion trajectories during synchronization tapping and found that asynchronies were smaller when a local minimum in height above the tapping surface subdivided the interval between taps. They proposed that the MNA occurs because participants underestimate the duration of empty intervals, and that auditory or motor subdivisions can increase the accuracy of duration estimates by providing structure for those intervals.

Both auditory and motor subdivisions between tones commonly occur in music ensemble performance. A given interval can be subdivided by tones produced by

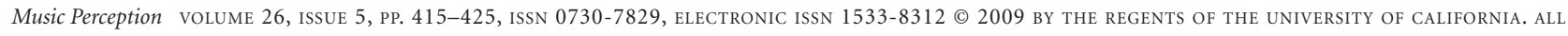
RIGHTS RESERVED. PLEASE DIRECT ALL REQUESTS FOR PERMISSION TO PHOTOCOPY OR REPRODUCE ARTICLE CONTENT THROUGH THE UNIVERSITY OF CALIFORNIA PRESS'S RIGHTS AND PERMISSIONS WEBSITE, HTTP://WWW.UCPRESSJOURNALS.COM/REPRINTINFO.ASP. DOI:10.1525/MP.2009.26.5.415 
another performer, in which case auditory information from the subdivision is available, or by the performer $\mathrm{him} /$ herself, in which case both auditory and movementrelated information are available. Previous findings indicate that the sensorimotor information arising from synchronized taps influences synchronization accuracy. The MNA is reduced when auditory feedback accompanies each tap in addition to the tactile and kinesthetic feedback already available (Aschersleben \& Prinz, 1995). The MNA is increased when tactile and kinesthetic feedback are reduced through weaker tapping movements with smaller amplitude or force (Aschersleben, Gehrke, \& Prinz, 2004) or eliminated through anesthesia (Aschersleben, Gehrke, \& Prinz, 2001). Although these findings, and the sensory accumulator model proposed to explain them (Aschersleben, 2002), are concerned only with the effects of feedback accompanying synchronized taps, they raise the possibility that synchronization accuracy might also differ depending on the types of sensory information that accompany subdivisions.

The current study addressed two questions. First, does sensorimotor information between tones in musical sequences influence pianists' ability to synchronize their performances with a metronome? Second, do these effects differ depending on whether the information is auditory, motor (movement-generated), or both? Experienced pianists performed melodies with a metronome. The task was manipulated so that auditory information, motor production, both, or neither occurred between synchronized tones. We hypothesized that both auditory information and motor production between tones would reduce the MNA, consistent with the tapping literature and the duration underestimation hypothesis proposed by Wohlschläger and Koch (2000). We also hypothesized that the combined auditory and motor information would reduce asynchronies more than either alone, given previous reports that providing auditory feedback in addition to motion-generated feedback on synchronized taps reduces the MNA. Wohlschläger and Koch (2000) attributed the reduced asynchrony when non-contact movements occurred between taps to changes in finger motion trajectories. It is possible that similar changes in motion trajectories might underlie increased synchronization accuracy when participants hear but do not have to produce subdivisions, particularly given the close link between auditory and motor networks in musicians (e.g., Haueisen \& Knösche, 2001). We therefore recorded and analyzed pianists' finger motions, and hypothesized that subdivision conditions that reduced asynchronies would also result in changed motion trajectories.

\section{Method}

Participants

One male and sixteen female pianists, ranging in age from 17 to 27 years $(M=20.59, S D=2.52)$, were recruited from the McGill community. All participants had at least 7 years of piano instruction $(M=11.06, S D=$ 2.80 ), and all but one were right-handed. ${ }^{1}$ All participants gave informed consent according to the procedures approved by the Institutional Review Board of McGill University and all procedures were consistent with the Helsinki Declaration.

\section{Stimulus Materials and Design}

Two sets of four two-measure melodies were designed for the study. The first measure of one set of melodies is shown in the left panel of Figure 1. Each melody contained eighth notes on all beats ( 1 beat $=1$ quarter note in all melodies). Within each set, one melody contained no subdivisions between quarter-note beats (labeled "NONE" in the figure and referred to as the no-subdivision melody in text); the others contained eighth notes subdividing odd beats (ODD), even beats (EVEN), or all beats (ALL). All melodies were written in $4 / 4$ meter and were performed with the right hand. One set of melodies was notated in the key of $\mathrm{C}$ major and the other in $\mathrm{G}$ major. Only the first five notes of each scale were used so that participants did not have to reposition their hands during performance. Beats were produced approximately equally often by each of the fingers and thumb.

Participants produced each notated melody while hearing one of the four melodies within each set, in a within-subjects design. This design is depicted schematically in Figure 1. Produced melodies appear on the left side of the figure and the associated auditory feedback on the right side. Participants completed the fully crossed design, which yielded 16 combinations of produced and heard melodies. Lines connecting the melodies in Figure 1 denote the 10 conditions in which all subdivisions resulted from motor production only, auditory information only, both, or neither (analyzed separately, below). In all cases where produced and heard notes coincided (for example, all quarter-note beats), participants heard the auditory feedback associated with the keystroke they produced. In the control condition,

\footnotetext{
${ }^{1}$ The pattern of results did not change when the left-handed participant was excluded from analysis; therefore, analyses included all seventeen participants.
} 


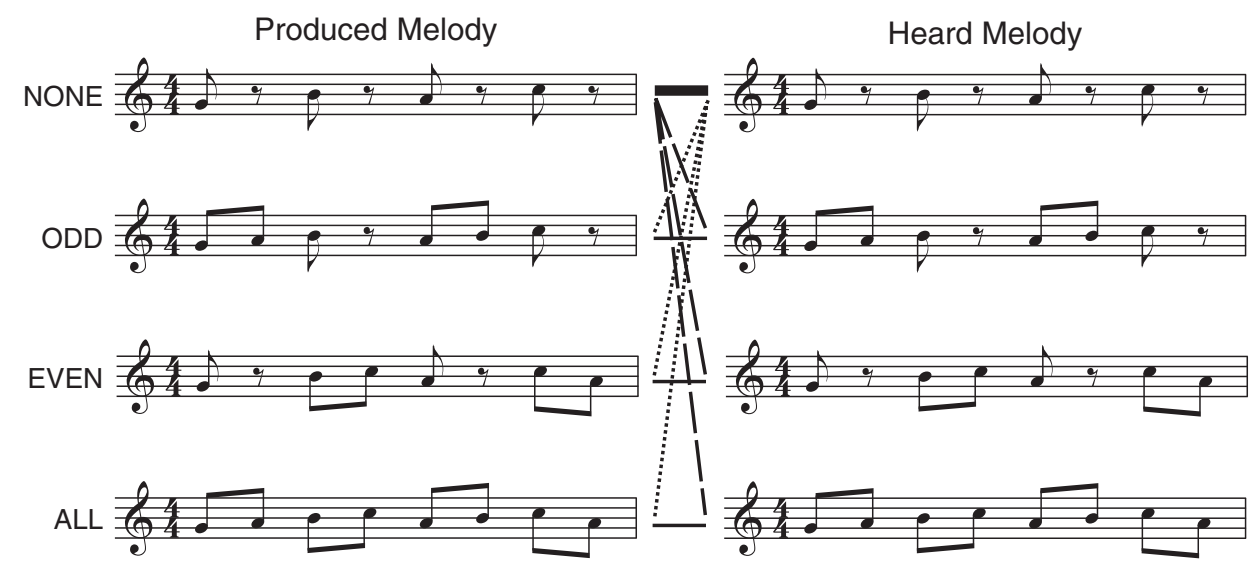

FIGURE 1. The first measure from one set of stimulus melodies. Melodies on the left were produced by participants while they heard auditory feedback corresponding to one of the melodies on the right. The thick black line connecting produced and heard melodies denotes the no-information condition. Dashed, dotted, and solid lines denote the auditory, motor, and auditory + motor information conditions, respectively.

denoted by the thick solid line, subdivisions were neither produced nor heard. This condition is referred to as the no-information condition; for ease of exposition, subdivision conditions are referred to by type of sensory information. In the three auditory + motor information conditions, denoted by thin solid lines, participants heard all of the subdivisions they produced (i.e., normal feedback). In the three motor information conditions, denoted by dotted lines, participants heard the nosubdivision melody while producing melodies with subdivisions (i.e., auditory feedback was removed from the subdivisions participants produced). In the three auditory information conditions, denoted by dashed lines, participants produced the no-subdivision melody while hearing melodies with subdivisions. Thus, participants heard auditory subdivisions between the quarter-note beats they produced. The timing of these auditory subdivisions was based on each participant's performance under normal feedback conditions, so that their subdivision timing would be approximately the same across all subdivision conditions. The timing, determined separately for each participant and set of melodies, was based on the mean duration of the interval from each beat to the following subdivision within one practice trial of the all-subdivision melody. Participants also performed the remaining six melody combinations; however, these conditions are of less interest because the sensorimotor information associated with the subdivisions was mixed within each sequence.

\section{Equipment}

The three-dimensional motion of each finger was recorded using Certus (Northern Digital Inc., Waterloo, Ontario, Canada) infrared-emitting diodes
(IREDs) at a sampling rate of $250 \mathrm{~Hz}$. Certus active sensors measured the position of the markers with a precision of $<0.1 \mathrm{~mm}$ at this sampling rate. Diodes were placed on the participant's right hand near the tip of each fingernail, on the skin to the right of the thumbnail, and on the head of the ulna (wrist). As the IREDs are small and lightweight, interference with performance was minimal.

Melodies were performed on a Roland RD-700SX (Roland Corporation, Los Angeles, CA, USA) weighted-key digital piano which gave the cameras an unobstructed view of the fingertips. Presentation of auditory feedback and metronome pulses, as well as MIDI data acquisition, was implemented via the FTAP software program (Finney, 2001). Timbres were generated by an Edirol StudioCanvas SD-80, using a piano timbre from the Contemporary bank (Rock Piano, Instrument \#002) for performances and a drum timbre from the Classical bank (Standard Set, Instrument $\# 001)$ for the metronome pulses. The participants heard performances and metronome pulses over AKGK271 headphones. MIDI velocity of all auditory feedback was held constant and the volume was adjusted to a comfortable level for each participant. The MIDI data subsequently were aligned with the motion data via the metronome pulse audio signal, which was recorded on both the analog input of the Certus system and the sound card that recorded the MIDI keystrokes.

\section{Procedure}

Participants were presented first with all four melodies from one set, which they practiced under normal feedback conditions until they could produce the melodies without error. Then they practiced synchronizing with 
the metronome set at $500 \mathrm{~ms}$ per quarter-note beat under normal feedback conditions and finally with altered feedback. On each experimental trial, four metronome beats were sounded and participants began performing along with the fifth beat. Participants were instructed to synchronize their performances with the metronome and to repeat the melody until the metronome stopped, which happened four beats after three repetitions of the melody (a total of 24 beats). Participants performed three blocks of 16 trials (4 produced melodies $\times 4$ heard melodies) for each of two sets of melodies, for a total of 96 trials. Each block of trials contained all 16 produced $\times$ heard melody combinations presented in pseudorandom order such that the same produced melody never occurred twice in a row. Participants also completed a questionnaire about their musical backgrounds. Participation in the experiment took approximately 1.5 hours, and participants received a nominal fee.

\section{Data Analysis}

The analyses reported here focus on timing and motion used to produce beats at the quarter-note level. A total of 16 on-beat keystrokes were included from the last two cycles of each performed melody per trial (the first repetition was excluded from analysis). Nine trials $(0.55 \%$ of total trials) were excluded from analysis due to pitch errors in the performed melodies. Timing analyses were based on MIDI onset times ( $1 \mathrm{~ms}$ temporal resolution). Interonset intervals (IOIs) were defined as the time interval between one MIDI onset and the MIDI onset that preceded it. The asynchrony for each beat was calculated by subtracting the metronome onset time from the MIDI onset time.

The motion analyses focus on finger motion in the z-plane (height above the keyboard), as the melodies required little movement in the $\mathrm{x}$ - and $\mathrm{y}$-planes. Sixteen keystrokes (less than $0.01 \%$ of the data) contained missing values due to occlusion factors and were excluded from analysis. Analyses of the finger motion trajectories were conducted using functional data analysis techniques (Ramsay \& Silverman, 2005). First, keystroke onsets in the motion data were identified by the peak in acceleration that occurred when the finger was stopped as the piano key arrived at the key bottom (Goebl \& Palmer, 2008). Next, order 6 B-splines were fit to the second derivative (acceleration) of the motion data using one knot for every five data points. The data were smoothed using a roughness penalty on the fourth derivative $\left(\lambda=10^{-18}\right)$, which allowed for control of the smoothness of the second derivative. The smoothed data then were interpolated to create 125 equally spaced observations between each keystroke onset; the acceleration curves were aligned (coregistered) across fingers in terms of the arrival time of the finger producing each keystroke. All motion trajectory analyses compared the equally spaced observations between on-beat keystrokes.

\section{Results}

Participants accurately produced the melodies at the rate set by the metronome. The mean interonset interval $(M=500.01 \mathrm{~ms}, S E=0.18)$ was equivalent to the prescribed metronome rate of $500 \mathrm{~ms} /$ beat. A one-way analysis of variance (ANOVA) on IOI by sequence position yielded no significant main effect, indicating that tempo drift did not occur within trials. A one-way ANOVA on the mean durations from each beat to the following subdivision (these durations were selfproduced in the motor and auditory + motor information conditions and produced by the computer in the auditory information conditions) revealed small but significant differences across conditions. Durations were shortest in the motor information condition $(M=$ $237.13 \mathrm{~ms} ; S E=0.93)$, longer in the auditory + motor condition $(M=240.20 ; S E=0.85)$, and longest in the auditory condition $(M=244.21 ; S E=0.50), F(2,32)=$ $34.59, p<.001$, Tukey's HSD $=2.10$. Because we primarily were interested in synchronization accuracy, the following analyses focus on asynchronies between onbeat keystroke onsets and the metronome, and on the finger motion trajectories immediately preceding the keystroke onsets.

\section{Mean Asynchrony Across Sequences}

We first analyzed mean signed asynchronies in a 4 (produced melody: none, odd, even, all) $\times 4$ (heard melody: none, odd, even, all) repeated-measures ANOVA, which revealed main effects of produced melody, $F(3,48)=$ $3.07, p<.05$, and heard melody, $F(3,48)=46.19, p<.001$, and a significant interaction, $F(9,144)=7.47, p<.001$. To assess whether the presence of sensory information between beats affected synchronization accuracy, we conducted posthoc tests contrasting performance in the no-information condition (labeled "NONE" in Figure 2) with performance in the conditions in which sensory information between beats was exclusively auditory, motor, or auditory + motor (see Design, above). Mean asynchronies for these ten conditions are shown in Figure 2. Stars above bars on the right indicate which conditions were significantly different from the no-information condition (leftmost bar), Tukey's HSD $=5.00, p<.05$. As the figure shows, 


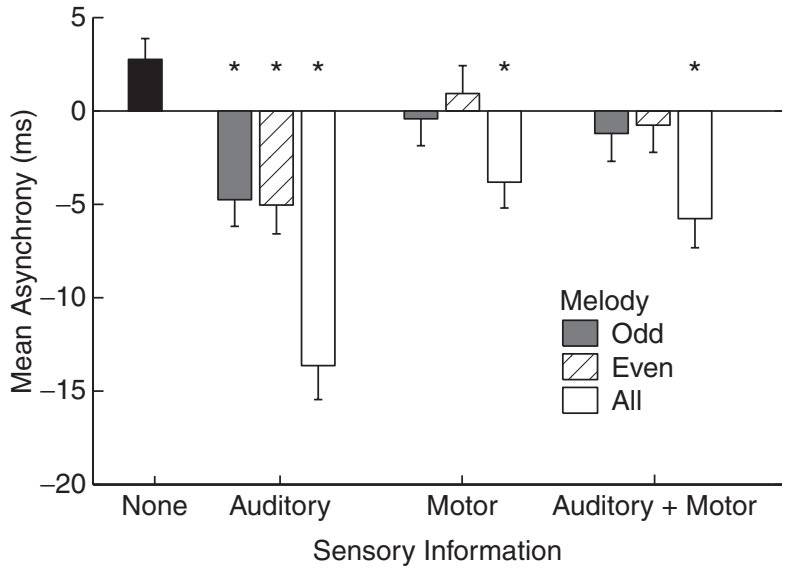

FIGURE 2. Mean asynchronies (+SE) for melodies with no sensory information between beats (leftmost bar) and with auditory, motor, or auditory + motor information between beats. Stars above bars on the right indicate which conditions were significantly different from the noinformation condition $(p<.05)$.

mean on-beat asynchrony occurred significantly earlier when auditory information occurred between beats than when no information occurred between beats, regardless of melody type (odd-, even-, or all-subdivision). Motor information and auditory + motor information between beats resulted in earlier asynchronies relative to the noinformation condition but only when information occurred between all beats of the melody (the all-subdivision melody). Comparisons within each melody type indicated that auditory information between beats resulted in significantly larger negative asynchronies than motor or auditory + motor information for all three melody types, Tukey's HSD $=5.00, p<.05$. Thus, participants produced beats earlier relative to the metronome when sensory information occurred between beats, particularly when that information was auditory.

We also conducted a 4 (produced melody: none, odd, even, all) $\times 4$ (heard melody: none, odd, even, all) ANOVA on the variance of asynchronies, as measured by the standard deviation of asynchronies within each trial. The ANOVA revealed a significant interaction, $F(9,144)=4.10, p<.001$, which was mainly due to smaller variance under normal feedback conditions relative to conditions in which feedback on subdivisions was altered. However, posthoc tests revealed that there were no significant differences between the ten conditions analyzed above.

\section{Mean Asynchrony by Sequence Position}

We next analyzed asynchronies at each sequence position within the melodies to examine the immediate impact of sensory information between beats. We were particularly interested in the pattern of asynchronies for the odd- and even-subdivision melodies because half of the beats were preceded by sensory information and the other half were not. Again we compared the melodies in which sensory information between beats was auditory, motor, or auditory + motor. An ANOVA by melody (odd- or even-subdivision), information type (auditory, motor, auditory + motor), and beat ( 1 to 4$)$ revealed a main effect of information type, $F(2$, $32)=9.80, p<.001$, as well as interactions between melody and beat, $F(3,48)=26.94, p<.001$, and melody, information type, and beat, $F(6,96)=4.74, p<.001$. Figure 3 shows the mean asynchronies for each beat within the odd- and even-subdivision melodies for auditory (top panel), motor (middle panel), and auditory + motor (bottom panel) information types. Beats that were preceded by sensory information (beats 2 and 4 for odd-subdivision melodies, beats 1 and 3 for evensubdivision melodies) were produced earlier relative to the metronome than beats that were not preceded by sensory information. Differences between asynchronies were significant for all beats when sensory information was auditory and for beats 2 and 4 when sensory information was motor, but were not significant when sensory information was auditory + motor, Tukey's HSD = $4.80, p<.05$.

We next compared asynchronies at each sequence position within the no-subdivision and all-subdivision melodies. This allowed us to determine whether asynchronies varied by beat independently of the alternating presence and absence of sensory information between beats. Within the all-subdivision melody, sensory information between beats could be auditory, motor, or auditory + motor; thus, we conducted an ANOVA by sensory information type (none, auditory, motor, auditory + motor) and beat (1 to 4$)$. The ANOVA revealed a main effect of information type, $F(3,48)=36.83, p<.001$, reflecting the pattern of larger negative asynchrony for melodies with sensory information between beats than melodies without, but no main effect of beat or interaction, $p s>.05$. This supports the conclusion that alternating asynchronies were specific to the melodies in which the presence and absence of between-beat sensory information alternated. Together with the results from the odd- and even-subdivision melodies, these results suggest that beats that were preceded by sensory information were produced earlier relative to the metronome than beats that were not preceded by sensory information.

To confirm this effect, we conducted an ANOVA on asynchronies in the odd-, even-, and all-subdivision 

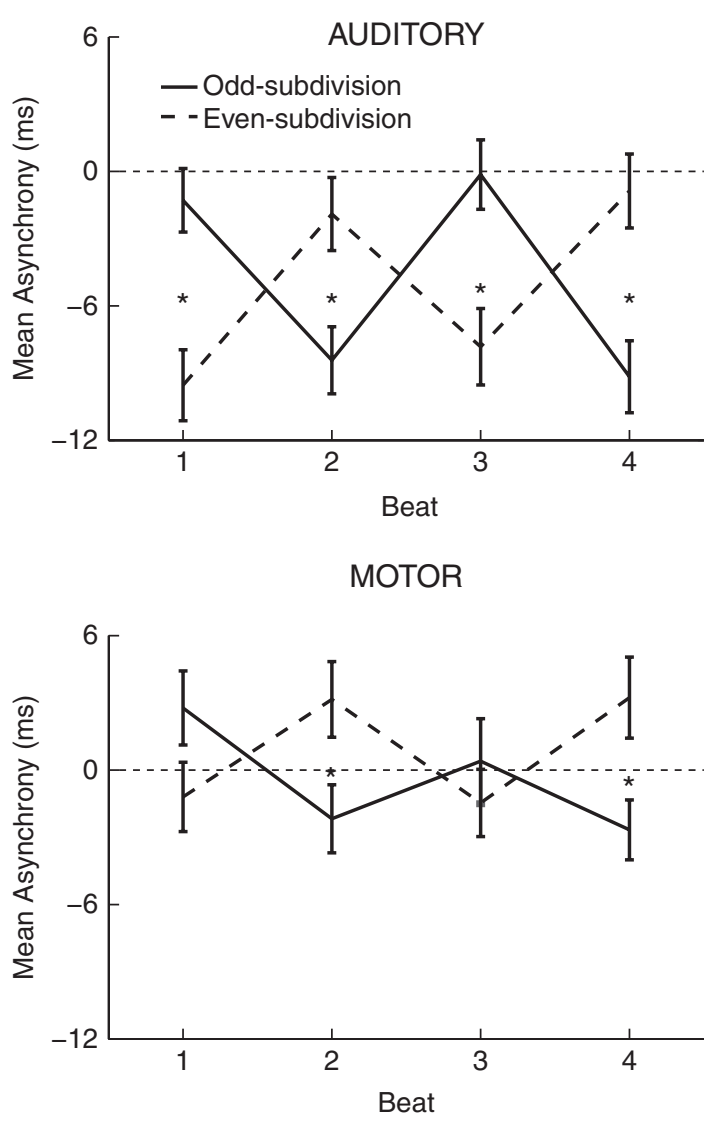

AUDITORY + MOTOR

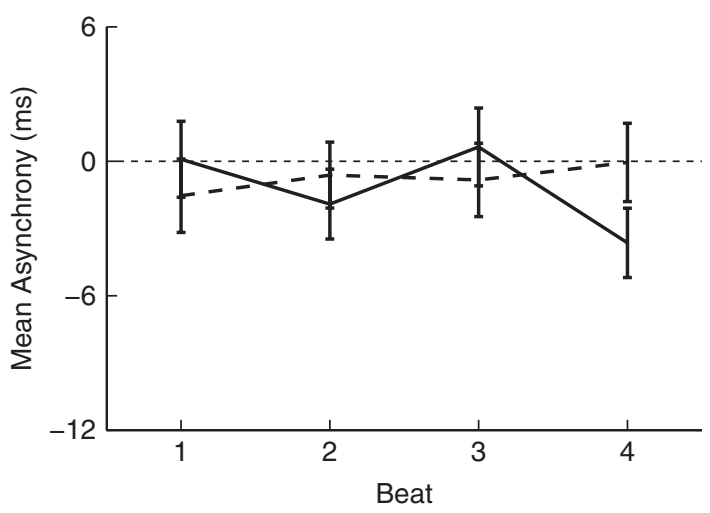

FIGURE 3. Mean asynchronies $( \pm S E$ ) by beat within the odd- and evensubdivision melodies for auditory (top panel), motor (middle panel), and auditory + motor (bottom panel) information. Stars indicate beats for which asynchronies differed significantly between melodies $(p<.05)$.

melodies by preceding sensory information (present, absent) and sensory information type (auditory, motor, auditory + motor). The ANOVA revealed main effects of preceding information, $F(1,16)=69.26, p<.001$, and information type, $F(2,32)=13.11, p<.001$, and an interaction, $F(2,32)=10.30, p<.001$. Posthoc comparisons indicated that beats that were preceded by sensory information were produced earlier relative to the metronome than beats that were not preceded by sensory information, when the information was auditory $[M s$ (SEs) $=-11.14$ (1.54) and -1.05 (1.41), Tukey's $\mathrm{HSD}=3.05]$, motor $[\mathrm{Ms}(\mathrm{SEs})=-2.85$ (1.26) and 2.34 (1.61)], and auditory + motor $[M s(S E s)=-3.88(1.43)$ and $0.01(1.52)$ ]. In sum, the analyses of mean asynchronies by sequence position confirmed the results that were documented across sequences: sensory information between beats increased negative asynchrony. Moreover, these analyses revealed that this occurred because beats that were preceded by sensory information were produced earlier relative to the metronome.

\section{Relationship Between Subdivision and Beat Timing}

We next examined the relationship between on-beat asynchrony and the timing of the immediately preceding sensory information to determine whether the impact of sensory information on asynchrony was related to the timing of the sensory information itself. The timing of sensory information between beats did not perfectly divide the metronome interval, because it was produced by participants themselves (motor and auditory + motor information) or based on participants' production under normal feedback conditions (auditory information). Sensory information between beats occurred an average of $13.12 \mathrm{~ms}(S E=0.68)$ before the midpoint of the metronome interval and did not differ across information conditions, $F(2,32)=$ $0.36, p>.05$. We calculated a "virtual asynchrony" between the observed onset of the sensory information and the expected midpoint of the metronome interval, and correlated this value (observed - expected) with the asynchrony of the immediately following beat. Across all melodies in which sensory information occurred between beats (odd-, even-, or all-subdivision melodies) and all sensory information conditions, all 17 participants showed a significant positive correlation between the virtual asynchrony of the sensory information and the produced asynchrony on the following beat, mean $r=.67$ (Fisher's $z^{\prime}$ transformed), all $p s<.001$. Thus, produced asynchronies were more negative when sensory information between beats occurred earlier than the expected midpoint between metronome pulses. We also computed the correlation separately for each combination of participant and sensory information type; mean correlations across participants were $r=.64$, $r=.69$, and $r=.66$ in the motor, auditory, and auditory + motor information conditions, respectively, all $p s<$ .001. Thus, the earlier the sensory information occurred 
relative to the midpoint between metronome pulses, the earlier the following beats were produced relative to the metronome.

\section{Motion Trajectories}

Finally, we examined whether sensory information preceding the beat influenced the motion used to produce the beat. Based on the timing results, we conducted functional ANOVAs (Ramsay \& Silverman, 2005) on the fingers' position and acceleration trajectories by preceding sensory information (present, absent) and sensory information type (auditory, motor, auditory + motor) to determine when the finger trajectories differed across conditions. Figure 4 shows the mean position (top half of each panel) and acceleration (bottom half) trajectories before keypresses that were and were not preceded by auditory (top panel), motor (middle panel), and auditory + motor (bottom panel) information, collapsed across odd-, even-, and all-subdivision melodies. In the figure, on-beat keypresses occurred at $0 \mathrm{~ms}$ and sensory information between beats occurred approximately $250 \mathrm{~ms}$ before.

The functional ANOVA on the position trajectories revealed a main effect of sensory information type, critical $F(2,32)=8.65, p<.001,{ }^{2}$ indicating that fingers were held higher above the keys when motor or auditory + motor information occurred between keypresses than when auditory information alone occurred between keypresses. There was also an interaction between information type and the presence of preceding sensory information, critical $F(2,32)=8.65, p<$ .001 . Posthoc tests were conducted within regions where the interaction reached significance; significant differences between trajectories that were and were not preceded by sensory information are indicated by horizontal brackets in the figure (critical $q$ for Tukey's HSD = $4.23, p<.01$ ). The figure shows that at the beginning of the trajectory, fingers were held higher above the keys when sensory information preceded the keypress than when sensory information did not precede the keypress, but only for motor or auditory + motor information. Examining the entire trajectory between keypresses reveals that a more pronounced local minimum

\footnotetext{
${ }^{2}$ The functional ANOVA yields an $F$-value as a function of time for each main effect and interaction. Effects were deemed significant when they exceeded the threshold $F$-value for the corresponding degrees of freedom. A conservative threshold of $p<.001$ was used to adjust for the multiple comparisons (Loehr \& Palmer, 2007; Vines, Krumhansl, Wanderley, \& Levitin, 2006).
}

occurred between keypresses when motor or auditory + motor information preceded the keypress than when sensory information did not precede the keypress. There was no evidence that auditory information alone influenced any portion of the finger position trajectories.

Acceleration trajectories also revealed an interaction between information type and presence of preceding information, critical $F(2,32)=8.65, p<.001$. Significant differences between trajectories that were and were not preceded by sensory information (critical $q$ for Tukey's $\mathrm{HSD}=4.23, p<.01)$ are again indicated by horizontal brackets beneath trajectories. Consistent with the position data described above, there were no differences between acceleration trajectories when the sensory information between beats was auditory. In contrast, differences between trajectories occurred approximately $250 \mathrm{~ms}$ before the on-beat keypress when the sensory information between beats was motor or auditory + motor. Both the motor and auditory + motor information conditions required the participant to produce a movement between keypresses; differences in acceleration trajectories occurred around the time this movement was produced. Acceleration remained at zero when no movement was required, but fluctuated around zero when movement was required. This pattern is consistent with biomechanical effects of other fingers' movements on acceleration trajectories in sequential finger movement tasks (Loehr \& Palmer, 2009).

\section{Discussion}

The current study examined the effects of different types of sensory information on synchronization performance. Normal solo performance usually yields coupled auditory and motor information from one's own feedback. In contrast, ensemble performance yields auditory information that can occur in the presence or absence of motor production. We used a paradigm that decoupled the auditory and motor information available to pianists who synchronized their performances with a metronome, to study the differential influences of auditory and motor information on their synchronization abilities. Both auditory information and motor production that occurred between quarter-note beats influenced pianists' ability to synchronize with those beats. Heard (auditory) and produced (motor) subdivisions increased the mean negative asynchrony; quarter notes that followed subdivisions were produced earlier than quarter notes that did not follow subdivisions. Pianists were sensitive to the timing of the subdivisions; sensory information that occurred earlier relative to the midpoint between 

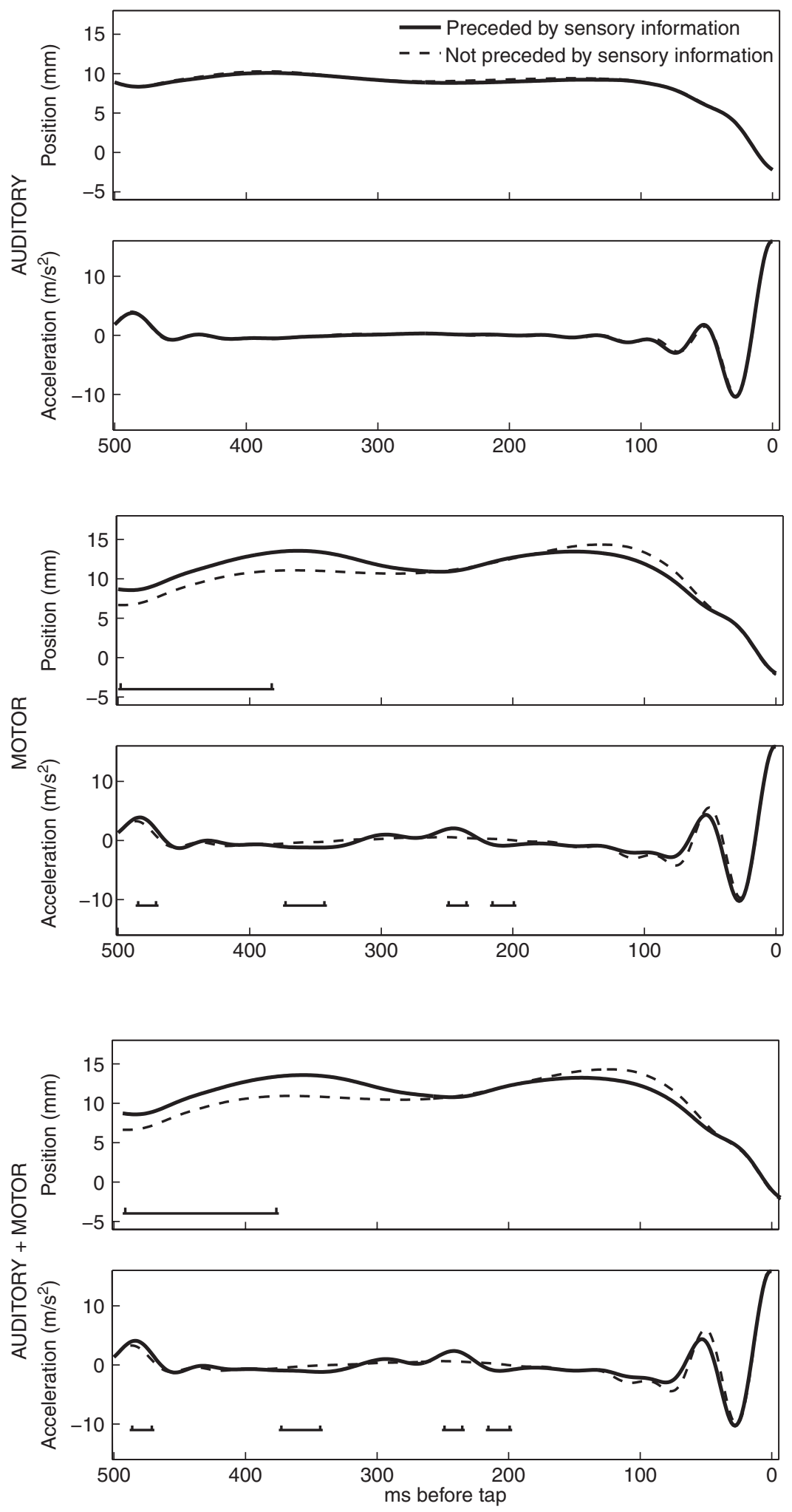

FIGURE 4. Mean position (top half of each panel) and acceleration (bottom half) trajectories before beats that were and were not preceded by auditory (top panel), motor (middle panel), and auditory + motor (bottom panel) information. On-beat keypresses occurred at 0 ms and sensory information between beats occurred approximately $250 \mathrm{~ms}$ before. Horizontal lines indicate where differences between trajectories reached significance $(p<.01)$. 
metronome pulses was associated with earlier production of the following beat. Auditory information placed between beats had a larger impact on the timing of performance than did motor production. In contrast, motor production between beats influenced finger motion trajectories, whereas auditory information did not.

Auditory information and motor production between beats decreased participants' temporal synchrony with a metronome. This contrasts with tapping studies in which subdivisions presented within a pacing (metronome) sequence or produced within a tapped sequence increased participants' synchronization accuracy or interonset timing accuracy, even if those subdivisions did not evenly divide the metronome interval (Pressing, 1998; Snyder, Hannon, Large, \& Christensen, 2006; Thaut et al., 1997; Wohlschläger \& Koch, 2000). This finding is also inconsistent with the perceptual underestimation hypothesis (Wohlschläger \& Koch, 2000). According to this hypothesis, subdivisions provide additional structure to unfilled intervals between synchronized taps, which assists with accurate estimation of temporal intervals and thereby reduces asynchronies. In the current study, sensory information that occurred earlier with respect to the midpoint between metronome beats was associated with earlier (less accurate) production of the immediately following beat. Similar to effects of auditory feedback on temporal relationships between produced and perceived timing in music performance (Pfordresher \& Palmer, 2002), this finding suggests that participants are sensitive to the timing of subdivisions, at least when they occur in the context of musical melodies. Thus, the presence of subdivisions alone-whether heard or produced-is not sufficient to increase the accuracy of synchronization in music performance.

Sensitivity to the timing of subdivisions is consistent with the phase correction response described by Repp $(2001,2002)$, in which tappers respond to a perturbation in a pacing sequence by shifting the timing of the following tap in the same direction as the perturbation. The phase correction response occurs even when the perturbed tones are subdivisions of the pacing tones with which participants must synchronize (Repp, 2008). Pianists' responses to asynchrony between heard or produced subdivisions and the midpoint of the metronome interval may be similar to participants' responses to perturbations in a pacing sequence. This seems particularly likely for auditory subdivisions that were not self-produced. Participants may have responded to these subdivisions as though they were part of the pacing sequence, despite instructions to synchronize with the metronome (which was a different timbre than the subdivisions). Auditory subdivisions might have increased synchronization accuracy if they had occurred at the midpoint between metronome intervals. However, subdivisions that perfectly divide the beat would not necessarily occur in ensemble performance, given that solo performers produce subdivisions earlier than the midpoint between beats when tapping rhythms or performing melodies (Gabrielsson, 1974; Gabrielsson, Bengtsson, \& Gabrielsson, 1983).

Motor and auditory + motor information between beats also resulted in shifted timing of the following beat in the current study, although this effect was smaller than that caused by auditory information between beats. Pianists may have responded to the early timing of their self-produced subdivisions with a smaller phase correction response than when computer-generated auditory subdivisions occurred between beats. Phase correction in the presence of self-produced subdivisions is consistent with previous findings indicating that the phase correction response is involuntary and difficult to suppress (Repp, 2002). Phase correction response studies usually perturb the timing of a single tone within the pacing sequence (Repp, 2001, 2002, 2008); in the current study, subdivisions with shifted timing occurred throughout the entire melody. Thus, pianists responded to timing shifts even when they occurred repeatedly across a sequence of events, and were part of the produced sequence rather than a pacing sequence with which the performer must synchronize.

Pianists' finger motions revealed different effects of sensory information between beats than did their timing data. Motor and auditory + motor information changed finger motion trajectories, whereas auditory information alone did not. Several aspects of the data suggest that the changes in finger motion caused by motor and auditory + motor subdivisions were due to biomechanical constraints of coupling between fingers. First, motion trajectories were altered only when participants had to produce an extra keypress movement between on-beat keypresses. Second, changes in finger position and acceleration trajectories were evident primarily at the time when a subdivision keypress was produced (around $250 \mathrm{~ms}$ before the beat). Third, changes in the motion of the finger producing the onbeat keypress were consistent with changes in motion used to demonstrate that a given finger's motion trajectory during another finger's tap is similar to the tapping finger's trajectory, due to biomechanical coupling between fingers (Li, Dun, Harkness, \& Brininger, 2004; Loehr \& Palmer, 2007, 2009). 
Auditory information between beats did not influence finger motion trajectories. Wohlschläger and Koch (2000) suggested that reduced asynchronies were the result of changes in finger motion trajectories; we therefore hypothesized that participants might produce small movements between synchronized beats in an attempt to reduce asynchrony. They did not do so. Instead, motor production influenced motion trajectories because of biomechanical constraints of coupling between fingers. Task differences may be responsible for these contrasting findings: the current study required sequential finger movements, whereas Wohlschläger and Koch (2000) measured single-effector tapping, which does not impose biomechanical coupling constraints. Task constraints might also explain the small positive asynchrony in the no-subdivision condition, which contrasts with previous reports of negative asynchrony when participants tap along with a metronome (Aschersleben, 2002). The current task required multifinger sequential movements in the production of musical melodies, whereas synchronization studies typically employ single-finger tapping movements accompanied by single tones or no auditory feedback.

In sum, auditory information and motor production that occurred between beats in music performance reduced pianists' synchronization accuracy. Beats that followed subdivisions were produced earlier relative to a metronome than beats that did not follow subdivisions. Pianists responded to the timing of betweenbeat sensory information by shifting the arrival of the following beat. The decoupling of auditory information and motor production demonstrated that the influence of sensory information on the timing of performance depends on its modality. Synchronization accuracy was affected most by auditory information but was also influenced by motor production. In contrast, motion trajectories were influenced only by motor production, due to biomechanical constraints on sequential finger motion.

\section{Author Note}

This research was supported in part by an NSERC Canada Graduate Scholarship to the first author and by the Canada Research Chairs program and NSERC Grant 298173 to the second author.

Correspondence concerning this article should be addressed to Prof. Caroline Palmer, Department of Psychology, McGill University, 1205 Dr. Penfield Avenue, Montreal QC H3A 1B1 Canada. E-MAIL: caroline. palmer@mcgill.ca

\section{References}

Aschersleben, G. (2002). Temporal control of movements in sensorimotor synchronization. Brain and Cognition, 48, 66-79.

Aschersleben, G., Gehrke, J., \& Prinz, W. (2001). Tapping with peripheral nerve block: A role for tactile feedback in the timing of movements. Experimental Brain Research, 136, 331-339.

Aschersleben, G., Gehrke, J., \& Prinz, W. (2004). A psychophysical approach to action timing. In C. Kaernbach, E. Schröger, \& H. Müller (Eds.), Psychophysics beyond sensation: Laws and invariants of human cognition (pp. 117136). Mahwah, NJ: Erlbaum.

Aschersleben, G., \& Prinz, W. (1995). Synchronizing actions with events: The role of sensory information. Perception and Psychophysics, 57, 305-317.

Finney, S. A. (2001). FTAP: A linux-based program for tapping and music experiments. Behavior Research Methods, Instruments, and Computers, 33, 65-72.

Gabrielsson, A. (1974). Performance of rhythm patterns. Scandinavian Journal of Psychology, 15, 63-72.

Gabrielsson, A., Bengtsson, I., \& Gabrielsson, B. (1983). Performance of musical rhythm in $3 / 4$ and $6 / 8$ meter. Scandinavian Journal of Psychology, 24, 193-213.
Goebl, W., \& Palmer, C. (2008). Tactile feedback and timing accuracy in piano performance. Experimental Brain Research, 186, 471-479.

Goebl, W., \& Palmer, C. (2009). Synchronization of timing and motion among performing musicians. Music Perception, 26, 427-438.

Haueisen, J., \& KnÖsche, T. R. (2001). Involuntary motor activity in pianists evoked by music perception. Journal of Cognitive Neuroscience, 13, 786-792.

Li, Z. M., Dun, S., Harkness, D. A., \& Brininger, T. L. (2004). Motion enslaving among multiple fingers of the human hand. Motor Control, 8, 1-15.

Loenr, J. D., \& Palmer, C. (2007). Cognitive and biomechanical influences in pianists' finger tapping. Experimental Brain Research, 178, 518-528.

Loehr, J. D., \& Palmer, C. (2009). Sequential and biomechanical factors constrain timing and motion in tapping. Journal of Motor Behavior, 41, 128-136.

Pfordresher, P. Q., \& PAlmer, C. (2002). Effects of delayed auditory feedback on timing of music performance. Psychological Research, 16, 71-79.

PREsSing, J. (1998). Error correction processes in temporal pattern production. Journal of Mathematical Psychology, 42, 63-101. 
Ramsay, J. O., \& Silverman, B. W. (2005). Functional data analysis (2nd ed.). New York: Springer.

Repp, B. H. (2001). Phase correction, phase resetting, and phase shifts after subliminal timing perturbations in sensorimotor synchronization. Journal of Experimental Psychology: Human Perception and Performance, 27, 600-621.

REPP, B. H. (2002). Automaticity and voluntary control of phase correction following event onset shifts in sensorimotor synchronization. Journal of Experimental Psychology: Human Perception and Performance, 28, 410-430.

REPP, B. H. (2003). Rate limits in sensorimotor synchronization with auditory and visual sequences: The synchronization threshold and the benefits and costs of interval subdivision. Journal of Motor Behavior, 35, 355-370.

Repp, B. H. (2005). Sensorimotor synchronization: A review of the tapping literature. Psychonomic Bulletin and Review, 12, 969-992.

Repp, B. H. (2006). Musical synchronization. In E. Altenmüller, M. Wiesendanger, \& J. Kesselring (Eds.), Music, motor control, and the brain (pp. 55-76). Oxford, UK: Oxford University Press.

Repp, B. H. (2008). Multiple temporal references in sensorimotor synchronization with metrical auditory sequences. Psychological Research, 72, 79-98.

Snyder, J. S., Hannon, E. E., Large, E. W., \& Christensen, M. H. (2006). Synchronization and continuation tapping to complex meters. Music Perception, 24, 135-146.

Thaut, M. H., Rathbun, J. A., \& Miller, R. A. (1997). Music versus metronome timekeeper in a rhythmic motor task. International Journal of Arts Medicine, 5, 4-12.

Vines, B. W., Krumhansl, C. L., Wanderley, M. M., \& Levitin, D. J. (2006). Cross-modal interactions in the perception of musical performance. Cognition, 101, 80-113. Wohlschläger, A., \& КосH, R. (2000). Synchronization error: An error in time perception. In P. Desain \& L. Windsor (Eds.), Rhythm perception and production (pp. 115-127). Lisse, The Netherlands: Swets \& Zeitlinger. 
